\title{
Backbone Chemical Composition and Monomer Sequence Effects on Phenylene Polymer Persistence Lengths
}

\author{
Nancy C. Forero-Martinez, ${ }^{* \dagger \dagger}{ }^{\dagger}$ Björn Baumeier, ${ }^{\ddagger}$ and Kurt Kremer* ${ }^{* \dagger \odot}$ \\ ${ }^{\dagger}$ Max Planck Institute for Polymer Research, Ackermannweg 10, 55128 Mainz, Germany \\ ${ }^{\ddagger}$ Department of Mathematics and Computer Science and Institute for Complex Molecular Systems, Eindhoven University of \\ Technology, P.O. Box 513, 5600 MB Eindhoven, The Netherlands
}

Supporting Information

ABSTRACT: Despite a vast body of the literature devoted to the use of phenylene polymers in the fabrication of graphene nanoribbons, the study of the physical properties of these precursors still poses open questions whose answers will certainly contribute to the design of more efficient/precise synthesis protocols. Particularly, persistence length measurements combined with size exclusion chromatography techniques assign both semiflexible to semirigid structures depending on the molecular weight of the precursor (Narita et al. Nat. Chem. 2014, 6, 126-132). Peculiarly, these results suggest an apparent

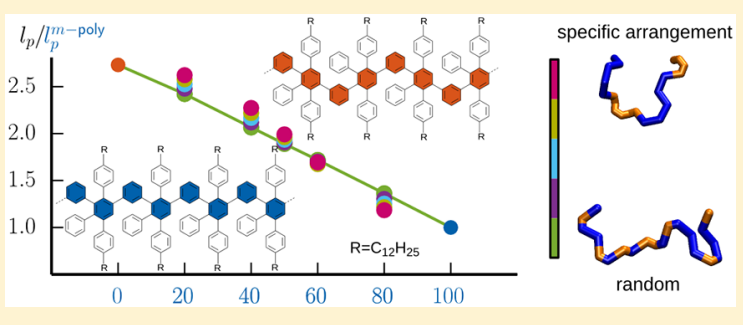
structural change upon increasing the length of the polymers. To address this puzzle, we use single-chain models to study the stiffness of polyphenylene precursors in a theta-like solvent as a function of chain composition and monomer sequence. Steric effects are isolated by considering random walk chains with segment length distributions and the position of monomers determined by the nature of the arene substitution along the backbone. Moreover, two homopolymer limiting cases are defined, that is, meta and para sequences, by associating two types of monomers to each possible substitution pattern. We consider, within these two limiting cases, chains with different compositions and monomer sequences. We compute persistence lengths, mean square end-to-end distances, and gyration and hydrodynamic radii. We find that distinct values of the persistence length for apparently the same chain chemistry are the result of different mixing ratios and the arrangement along the chain of the two positional isomers of the same monomer. Finally, we discuss the relation between two-dimensional density of the number of crossings and the length of polyphenylene segments as they would occur upon strong chain adsorption onto a substrate.

\section{INTRODUCTION}

Novel bottom-up techniques for the fabrication of graphene nanoribbons (GNRs) guarantee control of the produced structures with atomistic precision and therefore pave the way for designing GNRs with well-defined optoelectronic properties. ${ }^{2}$ As a matter of fact, long GNRs with various widths and edge structures have been obtained using solution-mediated synthesis. ${ }^{1}$ However, the tendency of long GNRs to aggregate, as evidenced by their poor solubility, prevents an accurate physical characterization and visualization and makes their deposition difficult. By contrast, surface-assisted methods to produce GNR structures enable their in situ visualization in combination with scanning tunneling microscopy $(\mathrm{STM})^{3}$ allowing a detailed experimental identification. In this protocol, polymerization and cyclodehydrogenation are both assisted by the metal surface such as $\mathrm{Au}(111)$ or $\operatorname{Ag}(111)$. However, this method has a low yield of GNRs of limited length. Not surprisingly, the deposition of GNRs is still a subject of intense investigation as revealed by an increasing number of publications devoted to such studies. $^{4-6}$ In particular, recent investigations report the successful deposition of isolated ribbons, produced via solution-mediated methods, on a treated $\mathrm{Si} / \mathrm{SiO}_{2}$ substrate ${ }^{4,5}$ and a reliable full layer transfer protocol of ribbons obtained by surface-assisted methods to an insulated substrate. ${ }^{6}$
In particular, long ( $>200 \mathrm{~nm})$ GNRs have been fabricated from tailor-made polyphenylene precursors using a solutionmediated approach. ${ }^{1}$ These precursors are produced through the AB-type Diels-Alder polymerization of small nonsymmetrical monomers, which induces positional isomerism, that is, two repeat units can be connected in two different ways depending on the two possible orientations of the monomers. ${ }^{1}$ Therefore, the resulting polymeric precursors are a combination of monomers connected in any of the positional isomerisms. Nonetheless, the resulting GNR after cyclodehydrogenation (graphitization and planarization) is unique, meaning that independent of the combination of monomers, the same GNR structure is obtained. In an attempt to increase the length of the produced GNR, two polyphenylene systems with different molecular weights were synthesized following the same synthesis protocol and characterized using static and dynamic light scattering ${ }^{2,7}$ and size exclusion chromatography (SEC). Surprisingly, large persistence length (Kuhn length) values, $l_{\mathrm{p}}=$ $9 \mathrm{~nm}\left(l_{\mathrm{k}}=18 \mathrm{~nm}\right)$, are reported. Furthermore, SEC analysis against polystyrene and poly( $p$-phenylene) standards gives

Received: April 25, 2019

Published: July 9, 2019 
characteristic semirigid and semiflexible polymeric structures for low- and high-molecular-weight precursors, respectively.

Persistence length measurements are key to characterize these phenylene polymers; however, these values do not take into account the monomer positional isomerism, which might be related to a different structure. Unfortunately, there is a lack of knowledge of how the polymer has been formed when obtaining/measuring persistence lengths in polyphenylenes.

We use computer simulations to investigate this conundrum. Since the size of the systems prevents a systematic approach by atomistic simulations, we resort to generic polymer models to obtain the backbone contribution to the persistence length. Particularly, single ideal chain models are a simple scheme aiming at studying conformational changes of polymer systems. An additional advantage of such models is that they are versatile enough to produce a large variety of conditions related to the chemical content and structure by changing a limited amount of parameters. While absolute numbers might not be accurate, we expect ratios of persistence lengths to be rather reasonably reproduced by such an approach. Here, we present a systematic study of the stiffness of polyphenylene precursors in a theta-like solvent as a function of chain composition and sequence of monomers. We build random walk chains with segment lengths and the position of monomers determined by the nature of the arene substitutions along the backbone, thus isolating their steric effects. In our model, we so far neglect excluded volume effects and keep the always present backbone steric effect that plays the role of a prefactor. Two types of monomers are associated to each of the two possible substitution patterns. In this manner, two homopolymer limiting cases are defined: polyphenylene formed exclusively of meta ( $m$-poly) or para ( $p$-poly) sequences. In between these limiting cases, we also consider chains with different compositions and monomer sequences.

In this work, we investigate the contribution of steric effects of the backbone to the persistence length for two selected polyphenylene precursors of graphene nanoribbons. ${ }^{1}$ To characterize the resulting chains, we compute persistence lengths, mean square end-to-end distances, and gyration and hydrodynamic radii. We show that different values of the persistence length for apparently the same chain chemistry with different molecular weights are the result of different arrangements along the chain of the positional isomerism of the same monomer. Finally, we discuss single-chain $2 \mathrm{D}$ projections onto a plane as if a chain was strongly adsorbed onto a surface. Such analysis could shed light on the possibility of the on-surface cyclodehydrogenation of the solution-synthesized polyphenylene precursors, which, to the best of our knowledge, has not yet been considered. Since on-surface polymerization fails to provide long GNRs thus far, ${ }^{3}$ an interesing alternative could be related to on-surface graphitization of solution-synthesized high-molecular-weight polyphenylene precursors. Furthermore, these $2 \mathrm{D}$ projection results are then related to the length of the polymer precursors and therefore to the efficiency of the polymerization for GNR synthesis.

\section{MODEL AND METHODS}

Linear polymer chains are represented as a succession of $(N+1)$ beads connected by $N$ bonds. Different geometrical constraints can be considered when modeling a polymer chain, namely, the bond length, $l_{\mathrm{b}}$, the angle between bonds, $\theta$, and an internal rotation angle (torsion angle rotation), $\phi$. This rotation is performed for the bead $i$ around an axis defined by the bond between beads $i-1$ and $i-2$.
Ideal chain models neglect interactions between monomers that are separated by a large number of bonds. However, different constraints can be taken into account to properly describe semiflexible polymers. In the freely jointed chain (FJC) model, bond lengths are fixed, and no correlation between bonds is assumed, meaning that all bond directions are equally probable and that bond and rotation angles around backbone bonds are chosen freely and independently of each other. In this model, the characteristic size of a polymer, given by the mean square end-to-end distance, is $\left\langle R_{\mathrm{e}}^{2}\right\rangle=N l_{\mathrm{b}}^{2}$. The freely rotating chain (FRC) model includes a restriction on the bond angles $\theta$, while keeping internal rotations free. Therefore, a correction due to the fixed bond angles is considered when calculating the square end-to-end distance, namely, $\left\langle R_{\mathrm{e}}^{2}\right\rangle=N l_{\mathrm{b}}^{2}(1+\cos \theta) /(1-\cos \theta){ }^{8}$

In real chains, internal rotation is prevented by the interaction between substituted side groups. This is the case of the hindered rotating chain (HRC) model, where bond lengths and bond angles are constant (fixed), and the internal rotation angle is chosen by a probability proportional to $\exp \left(-E(\phi) / k_{\mathrm{B}} T\right) . E(\phi)$ is the energy related to the torsion angle rotation, $k_{\mathrm{B}}$ is the Boltzmann constant, and $T$ is the temperature. As in the FRC model, a correction term, in this case, due to the hindered internal rotation appears in the mean square end-to-end distance ${ }^{9}\left\langle R_{\mathrm{e}}{ }^{2}\right\rangle=N l_{\mathrm{b}}{ }^{2}\left(\frac{1+\cos \theta}{1-\cos \theta}\right)\left(\frac{1+\langle\cos \phi\rangle}{1-\langle\cos \phi\rangle}\right)$, where

$$
\langle\cos \phi\rangle=\frac{\int_{0}^{2 \pi} \pi \cos \phi \exp \left(-E(\phi) / k_{\mathrm{B}} T\right) \mathrm{d} \phi}{\int_{0}^{2 \pi} \pi \exp \left(-E(\phi) / k_{\mathrm{B}} T\right)} \mathrm{d} \phi
$$

An equivalent freely jointed chain offers a unified characterization for ideal polymers. ${ }^{8}$ This equivalent chain has the same contour length $L$ and mean square end-to-end distance $\left\langle R_{\mathrm{e}}^{2}\right\rangle$ as the ideal chain. Then, for an equivalent chain with $N_{\mathrm{k}}$ bonds and effective Kuhn segment length $l_{\mathrm{k}}$,

$$
\left\langle R_{\mathrm{e}}{ }^{2}\right\rangle=l_{\mathrm{k}} L
$$

The Kuhn length, which is a way to measure the flexibility of a polymer chain, is related to the chain persistence length $l_{\mathrm{p}}$ by $l_{\mathrm{k}}=2 l_{\mathrm{p}}$. This condition holds in the random walk model and when $L \gg l_{\mathrm{k}}$.

The backbone structure contribution to the stiffness of the chain is well characterized by $l_{\mathrm{p}}$ along the chain, and it is computed within the HRC generic framework. Figure 1 sketches the repeat units (monomers) of these polymers. The difference lies in the way phenyl rings are connected to each other, either in the meta- $(\mathrm{m}-)$ or in the para-substitution $(p-)$.

We build ideal chains with different chemical structures, homo- and copolymers. In all cases, these chains correspond to random walks obtained by a Monte Carlo scheme and follow Gaussian statistics for large $L .{ }^{9}$ Each bead is located at the center of one of the backbone rings, and the angle $\theta$ between successive bonds is fixed $\left(\theta=120^{\circ}\right)$. The bond lengths, $l_{\mathrm{b}}^{m}, p$, correspond to the distances between two successive phenyl rings ( $m$-poly) and every other ring ( $p$-poly) along the backbone. This means that experimental monomers ${ }^{1}$ are represented by two beads in the meta-substitution pattern and by one bead in the para case. Nevertheless, when the data is analyzed, we evaluate the original contour length of the backbone, which corresponds to the molecular weight of the polymers. In practice, we construct the chain as follows: the positions of the three initial beads are arbitrarily chosen, thus defining a plane. The next bead is added at a fixed distance $l_{\mathrm{b}}$, angle $\theta$, and rotation angle $\phi$ with respect to the bond between beads 2 and 3 . The rotating bond then forms a circle in which the new bead lies. The position of this bead is related to the internal rotation angle, $\phi$, which turns out to be the torsion (dihedral) angle between two phenyl rings, and is selected with a probability proportional to the Boltzmann factor $\exp \left(-E(\phi) / k_{\mathrm{B}} T\right)$. This potential energy, $E(\phi)$, is the torsional potential between the two rings forming the backbone of the polymers. Therefore, $E(\phi)$ is obtained from scanning the dihedral angle defined by the four highlighted atoms in Figure 1. A single repeat unit, that is, two phenyl rings connected by a covalent bond and the corresponding side groups, is used to scan this dihedral angle for each studied system. Side chains are replaced by either hydrogen atoms, $\mathrm{CH}_{3}$ groups or benzene rings. Moreover, capping groups $\left(\mathrm{CH}_{3}\right.$ or benzene rings) are 


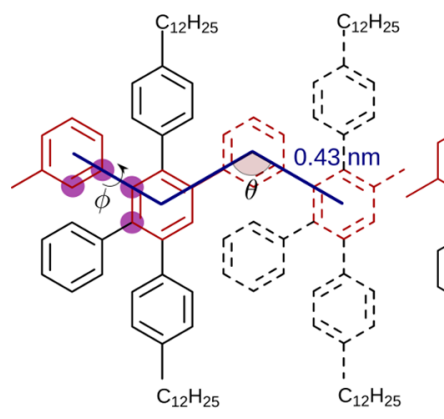

(a)

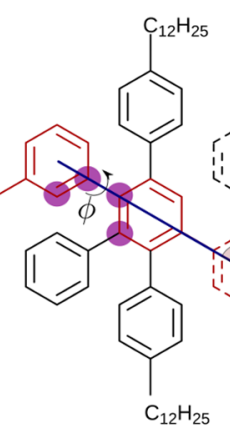

$\mathrm{C}_{12} \mathrm{H}_{25}$

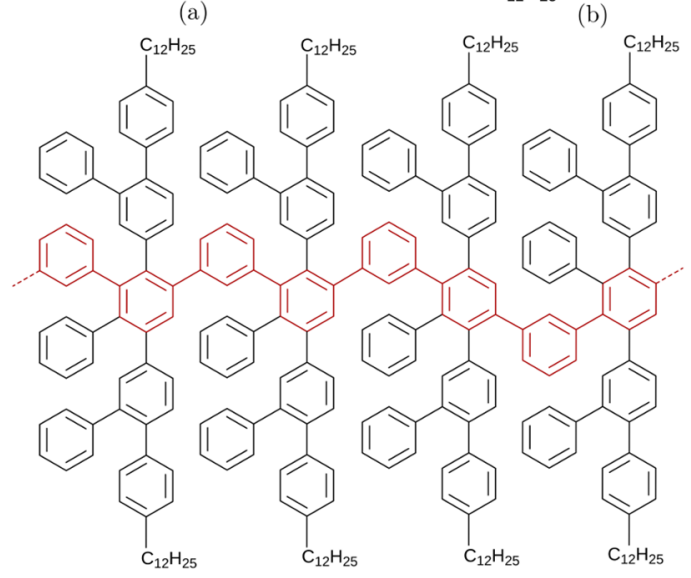

(c)
Figure 1. Schematic view of two repeat units for polymers (a) $m$-poly and (b) $p$-poly. The polymer backbone is shown in red (color online), with the angle between successive bonds $\theta=120^{\circ}$. Chains are characterized by the contour length $L=N l_{\mathrm{b}}$. The dihedral angle $\phi$ is defined by the four highlighted atoms in both cases. Extended lateral version (c) $m$ - and $p$-polymers, sharing the same backbone.

attached to the monomers. The upper panel in Figure 2 shows schematic representations for the different cases.

The potential energy scans are obtained by performing ab initio constrained geometry optimization for different values of the torsional angle $\phi$. The calculations were performed at the B3LYP/6-311 g(d,p) level of theory using the Gaussian software package. ${ }^{10}$ Figure 2 shows the potential energy curves in the case where side chains and capping groups are benzene rings for the two types of monomers. For comparison, we have also included the potential energy plots for the $m$ poly monomer when capping is made by $\mathrm{CH}_{3}$ groups, and side chains are either $\mathrm{H}, \mathrm{CH}_{3}$, or benzene rings. Symbols represent the original $\mathrm{ab}$ initio set of data, and lines correspond to polynomial interpolations. $m$ poly and $p$-poly interpolated energy values are the ones used to obtain the probabilities via the Boltzmann factor. We verify that, by increasing the complexity of the side groups, a large energy barrier arises as a dominant energy contribution due to the bulky decorating groups. Two phenyl rings with $\mathrm{H}$ atoms as side groups have a symmetric potential energy with maxima of the order of $2 \mathrm{kcal} / \mathrm{mol}$ and well-defined minima. When $\mathrm{CH}_{3}$ groups replace $\mathrm{H}$ atoms, the potential energy is characterized by a double-well potential with a large energy barrier. This qualitative picture does not change upon increasing the complexity of the side groups. However, the behavior of the minima depends on the particular side groups used. The structure in the potential energy surface appears as benzene rings replace $\mathrm{CH}_{3}$ groups. When capping groups are also changed by benzene rings, the rearrangement of the bulky groups with respect to each other leads to local minima and maxima. In Figure 2, the potential energies of the $m$-poly (blue) and $p$ poly (orange) systems exhibit small qualitative differences. This is a consequence of both the meta or para position of the connecting bond between monomers and the asymmetrical arrangement of the bulky groups with respect to each other upon the dihedral rotation in each
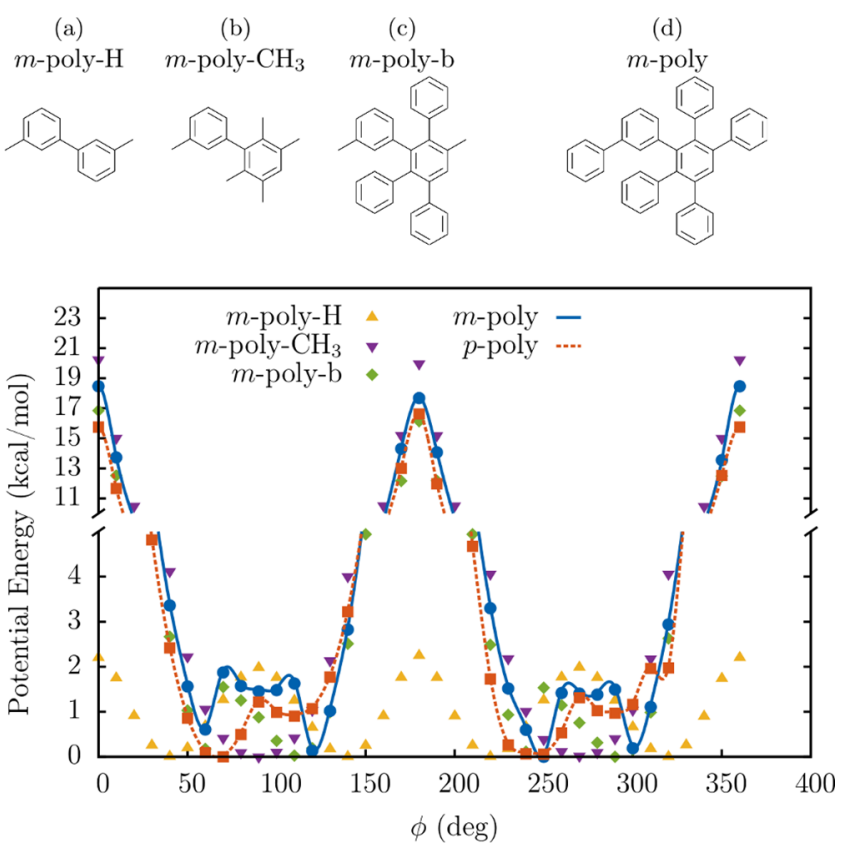

Figure 2. Potential energy scans for the dihedral angles defined in Figure 1. For $(\mathrm{a}-\mathrm{c}$, upper panels) $m$-poly-X systems, side chains are replaced by $\mathrm{X}=\mathrm{H}, \mathrm{CH}_{3}$ and $\mathrm{b}$ (benzene rings) with $\mathrm{CH}_{3}$ capping groups. Systems $m$-poly (d, upper panel) and $p$-poly have both capping and side groups replaced by benzene rings. The schematic twodimensional representations in (c) and (d) do not account for the molecule asymmetry. Symbols correspond to the original (from ab initio) data set, and lines represent the polynomial interpolations of the original data used in the Boltzmann factor computation.

case. We do not expect the $m$-poly and $p$-poly potential energy curves to present mirror symmetry. Figure S1 shows different energy contributions highlighting their impact on the total energy curve. As an example, Figures S3 and S4 show the behavior of some distances between atoms within the molecules and changes in the potential curves correlated to the loss of smothness of these curves. Figure 2 reveals another important feature, the energy barrier between minima states is between 15 and $20 \mathrm{kcal} / \mathrm{mol}$. Therefore, these states are way long lived and thus metastable, considering that the thermal energy at $300 \mathrm{~K}$ is only $0.6 \mathrm{kcal} / \mathrm{mol}$.

Homo- and copolymers are constructed as described above. However, when building random copolymers, different concentrations and sequence probabilities of the two phenylene monomers are taken into account. Changes in concentration are easily achieved by associating a probability $P_{\mathrm{t}}=P\left(l_{\mathrm{b}}^{\mathrm{t}}\right)(\mathrm{t}=m, p)$ to either type of substitution. Then, one Monte Carlo step consist of two parts: first, the type of monomer is selected according to the probabilities $P_{\mathrm{t}}$, thus determining the substitution pattern, and therefore the bond length and the torsional potential energy profile to be used; second, the position of the new bead is selected with probability $\exp \left(-E(\phi) / k_{\mathrm{B}} T\right)$. Even though the potential energy profiles do not show significant quantitative differences in their well values, little changes become important when looking at the Boltzmann probability when selecting the new bead position. It is clear that homopolymer configurations are obtained when the set of probabilities, $\left(P_{m}, P_{p}\right),(1,0)$ and $(0,1)$, are used. To consider a specific arrangement of the monomers along the chain, we include the conditional probability $P\left(l_{i}=l_{\mathrm{b}} \mid l_{i-1}=l_{\mathrm{b}}\right)$ of having a segment length $l_{i}=l_{\mathrm{b}}$ given that the previous segment length, $l_{i-1}$, is $l_{\mathrm{b}}$ (see the Supporting Information).

We consider chains lengths of up to $2 \times 10^{4}$ beads for two different "homopolymers" and five different compositions $(x, y)=\{(20,80)$, $(40,60),(50,50),(60,40),(80,20)\}$ or equivalently $\left(P_{m}, P_{p}\right)=$ $\{(0.2,0.8)(0.8,0.2)\} . x$ and $y$ correspond to the percentage of monomers $m$-poly and $p$-poly in the mixture. For each composition, five different conditional probabilities, from 0.5 to 0.9 , are used, where 
0.5 corresponds to a randomly organized chain and 0.9 corresponds to the case where an ordered sequence of monomers is favored. All results discussed here are averaged over $10^{4}$ independent configurations.

\section{RESULTS AND DISCUSSION}

Solution-mediated synthesis allows the production of a unique GNR structure from polyphenylene precursors. However, the monomer building block has two possible orientations, and therefore, polyphenylene precursors are the result of randomly connected positional isomers. ${ }^{1}$ Although, experimentally, the focus has been on the resulting GNR structures, being able to characterize the precursors based on their structural/chemical properties makes it possible to design GNRs with improved optoelectronic properties.

Bond correlations and connectivity within a chain can be described by the mean square internal distances, $\left\langle R^{2}(s)\right\rangle$, given by

$$
\left\langle R^{2}(s)\right\rangle=\frac{1}{M} \sum_{n=1}^{M}\left(\frac{1}{(N+1)-s} \sum_{i=1}^{(N+1)-s}\left(\mathbf{r}_{n, i+s}-\mathbf{r}_{n, i}\right)^{2}\right)
$$

where $\langle\cdots\rangle$ corresponds to the average over the ensemble of possible conformations, $M, N$ is the number of bonds in a chain, $s$ corresponds to the chemical distance, that is, the number of bonds between two beads, and $\mathbf{r}_{n, i}$ is the position vector of the bead $i$ in the chain $n$. Figure 3 shows the mean square internal

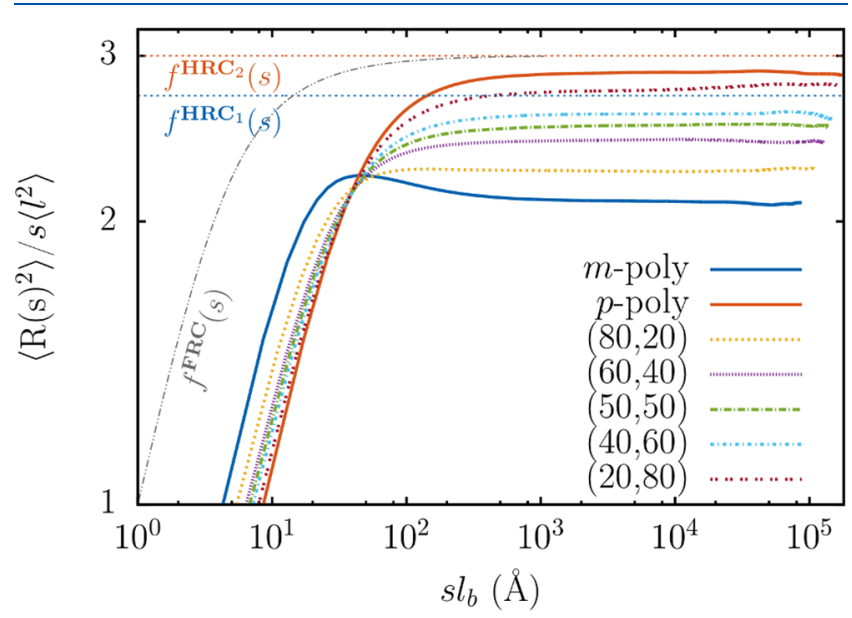

Figure 3. Mean square internal distance $\left\langle R^{2}(s)\right\rangle / s\left\langle l^{2}\right\rangle$ for homopolymers $m$-poly (solid blue line) and $p$-poly (solid orange line) and for copolymer chains with different concentrations $(x, y) . f^{\mathrm{FRC}}(s)$ corresponds to the theoretical prediction for an FRC, $f^{\mathrm{FRC}}(s)=\frac{1+\langle\cos \theta\rangle}{1-\langle\cos \theta\rangle}-\frac{2\langle\cos \theta\rangle\left(1-\langle\cos \theta\rangle^{s}\right)}{s(1-\langle\cos \theta\rangle)^{2}}$, and $f^{\mathrm{HRC}}(s)$ corresponds to the end-to-end distance in the HRC model, $f^{\mathrm{HRC}}(s)=\left(\frac{1+\cos \theta}{1-\cos \theta}\right)\left(\frac{1+\langle\cos \phi\rangle}{1-\langle\cos \phi\rangle}\right)$. The root mean square bond length is $l_{\mathrm{b}}=\sqrt{\left\langle l^{2}\right\rangle}$.

distance for the different systems under consideration at $T=300$ $\mathrm{K}$. Since we are imposing a fixed bond angle, we include the corresponding theoretical prediction for the freely rotating chain, $f^{\mathrm{FRC}}(s)$, that takes into account the correlations between bond directions. ${ }^{9}$ In addition, we also plot the corresponding expression for the HRC in the limit of large $N$ in the homopolymer cases $\left(f_{(1,2)}^{\mathrm{HRC}}(s)\right)$.

Curves representing randomly mixed chains are between the two homopolymer limiting cases: $m$-poly (lower value curve) and $p$-poly (higher value curve). At large $s l_{\mathrm{b}}$ values, $\left\langle R^{2}(s)\right\rangle / s\left\langle l^{2}\right\rangle$ reaches a constant value related to the persistence length of the chain. The average bond length, $l_{b}$, and therefore the contour length both increase as the composition of the chain goes from low to high $p$-poly monomer content. The crossing point of the curves roughly represents chains that have the same contour length given by $s l_{\mathrm{b}}$. Deviations from the FRC model are more notorious in the case of $m$-poly because of the interplay between the short length bond and constrained internal rotation angle.

Finite Size Effects. Another more global quantity to characterize the size of a linear polymer chain with contour length $L=l_{b} N$ is given analytically by the mean square end-toend distance in eq 2

$$
\left\langle R_{\mathrm{e}}{ }^{2}\right\rangle=\left\langle\left(\mathbf{r}_{n, N+1}-\mathbf{r}_{n, 1}\right)^{2}\right\rangle
$$

In addition, probability distributions of the different structural parameters are a natural way to describe the conformational behavior of single polymer chains. Figure 4 shows the theoretical

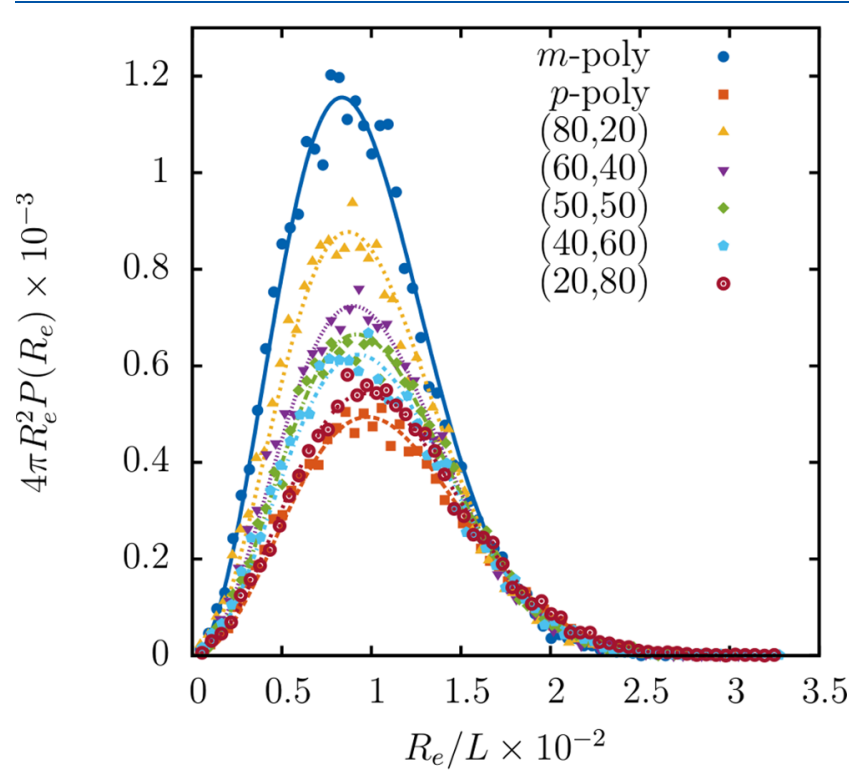

Figure 4. Probability distributions of the end-to-end distance for different chain compositions. Symbols correspond to the random walk probability distributions and are a measure of the quality of our data, while lines represent the theoretical distributions (eq 5).

distribution of the end-to-end distance (solid lines), $4 \pi R_{\mathrm{e}}^{2} P\left(R_{\mathrm{e}}\right)$, for ideal chains of different compositions compared to the probability distribution obtained from the random walk (symbols). This probability distribution $P\left(\vec{R}_{\mathrm{e}}\right)$ is defined as

$$
P\left(\vec{R}_{\mathrm{e}}\right)=\left(\frac{3}{2 \pi\left\langle R_{\mathrm{e}}{ }^{2}\right\rangle}\right)^{3 / 2} \exp \left(-\frac{3 \vec{R}_{\mathrm{e}}^{2}}{2\left\langle{R_{\mathrm{e}}}^{2}\right\rangle}\right)
$$

Moreover, the polymer coil size is well characterized by a structural physical quantity, the radius of gyration $R_{\mathrm{g}}$,

$$
\left\langle R_{\mathrm{g}}{ }^{2}\right\rangle=\frac{1}{(N+1)} \sum_{i=1}^{N+1}\left\langle\left(\mathbf{r}_{i}-\mathbf{r}_{\mathrm{cm}}\right)^{2}\right\rangle
$$

where $\mathbf{r}_{\mathrm{cm}}$ corresponds to the position of the center of mass, $\mathbf{r}_{\mathrm{cm}}=$ $\sum_{i=1}^{N+1} \mathbf{r}_{i} /(N+1)$. A third quantity, obtained from dynamic measurements, namely, the hydrodynamic radius $R_{\mathrm{h}}$, is defined as 

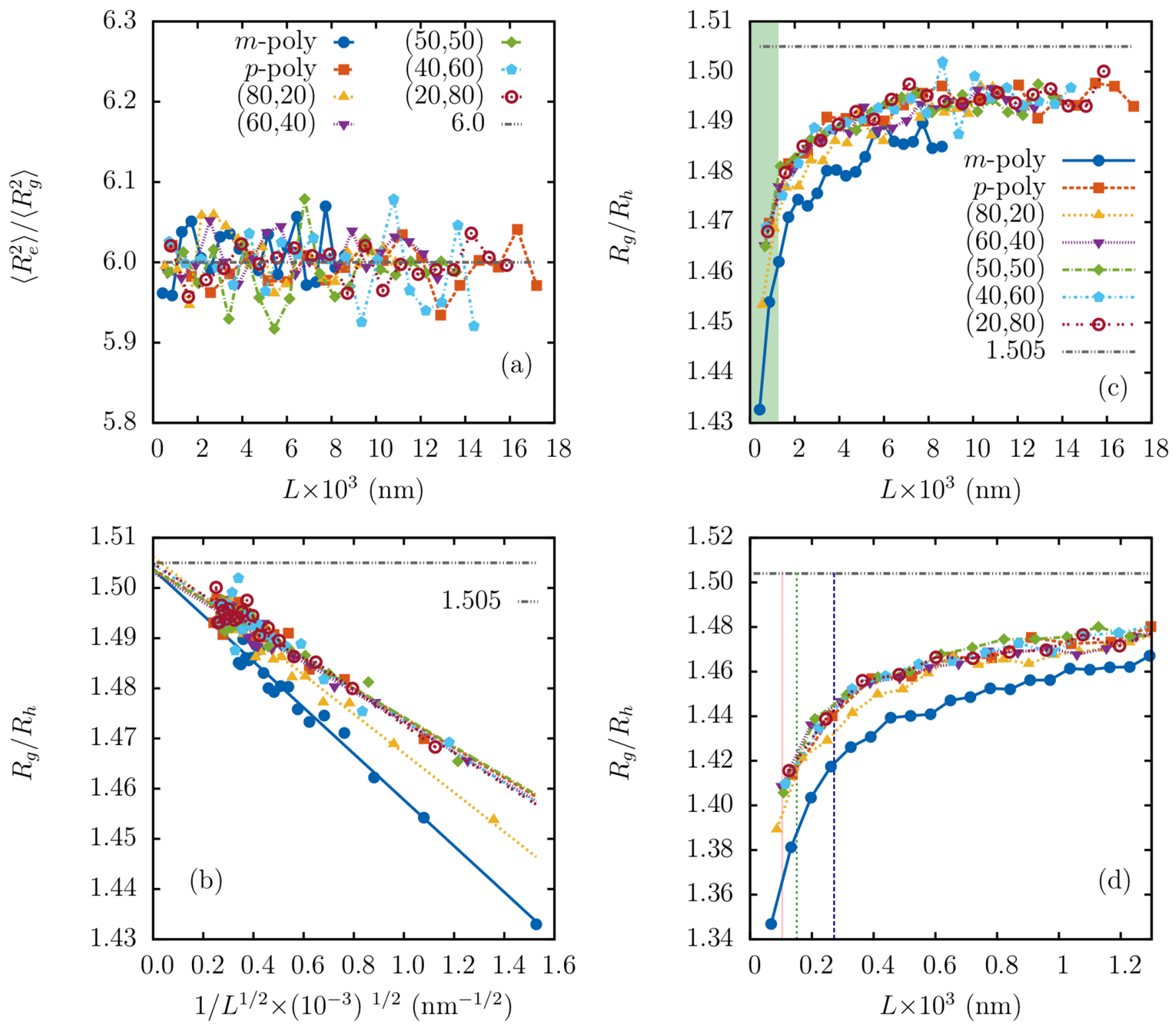

Figure 5. Ratio between (a) the mean square end-to-end distance and the mean square radius of gyration, $\left\langle R_{\mathrm{e}}^{2}\right\rangle /\left\langle R_{\mathrm{g}}^{2}\right\rangle$, and (b-d) the mean square radius of gyration and the hydrodynamic radius, $R_{\mathrm{g}} / R_{\mathrm{h}}$ for different fractions of meta and para units. The light green region in (c) corresponds to the range of experimental measurements, ${ }^{1}$ while (d) is a detailed plot of the calculated $R_{\mathrm{g}} / R_{\mathrm{h}}$ in this region. Vertical lines (solid pink, dotted green, and dashed blue) represent contour length values obtained from the $M_{\mathrm{W}}$ values of three polyphenylene precursor samples resulting from different preparation conditions. ${ }^{1}$ Experimental ratios are $R_{\mathrm{g}} / R_{\mathrm{h}}^{2-\mathrm{I}, 2-\mathrm{II}}=1.1428,1.3104$ for two isomeric mixtures 2-I and 2-II. ${ }^{1}$ Horizontal dashed lines correspond to the theoretical ratios for a polymer in a theta solvent, $\left\langle R_{\mathrm{e}}^{2}\right\rangle /\left\langle R_{\mathrm{g}}^{2}\right\rangle=6.0$ and $R_{\mathrm{g}} / R_{\mathrm{h}}=1.505$, in the $N \rightarrow \infty$ limit.

$$
\left\langle\frac{1}{R_{\mathrm{h}}}\right\rangle=\frac{1}{(N+1)^{2}} \sum_{\substack{m, n \\ m \neq n}}\left\langle\frac{1}{\left|\mathbf{r}_{m}-\mathbf{r}_{n}\right|}\right\rangle
$$

For an ideal chain $\left\langle R_{\mathrm{e}}^{2}\right\rangle \propto\left\langle R_{\mathrm{g}}^{2}\right\rangle \propto\left\langle R_{\mathrm{h}}^{2}\right\rangle \propto N, R_{\mathrm{h}}$ is smaller than $R_{\mathrm{g}}$ and $R_{\mathrm{e}}$, with theoretical ratios of $R_{\mathrm{h}} / R_{\mathrm{e}}=0.271$ and $R_{\mathrm{g}} / R_{\mathrm{h}}=$ 1.505 for a polymer in a theta-like solvent. ${ }^{11}$

Figure 5 shows the characteristic ratios for the different chain compositions and monomer sequences compare against theoretical values for ideal chains. The $\left\langle R_{\mathrm{e}}^{2}\right\rangle /\left\langle R_{\mathrm{g}}^{2}\right\rangle$ ratio lies in the asymptotic region even for short chains. However, significant finite size effects are observed for ratios of the hydrodynamic radius and both the end-to-end distance and the radius of gyration. By definition, $R_{\mathrm{h}}$ gives more weight to shorter distances resulting in a slow converging ratio; nevertheless, for longer chains, an asymptotic value is approached. In the $N \rightarrow \infty$ limit, the relation $R_{\mathrm{g}} / R_{\mathrm{h}}$ for a Gaussian chain follows ${ }^{12}$

$$
\frac{R_{\mathrm{g}}}{R_{\mathrm{h}}}=\frac{8}{3 \sqrt{\pi}}\left(1+B N^{-1 / 2}\right)
$$

where $B$ is a positive constant. In the case of self-avoiding walks, this universal ratio is somewhat larger and has been accurately estimated by high-resolution Monte Carlo techniques, reaching a value of $1.5803940(45),{ }^{13}$ surpassing previous estimated values. $^{12}$ Figure $5 \mathrm{~b}$ shows this ratio as a function of $1 / L^{1 / 2}$ (symbols) and a linear fit (lines) is used to find the asymptotic value for all chains. Figure $5 \mathrm{c}$ shows the ratio $R_{\mathrm{g}} / R_{\mathrm{h}}$ as a function of $L$ for different chain compositions. This ratio converges to 1.505 (horizontal dashed line) for all cases as the size of the chain increases. The light green region $(7.3<L<1278 \mathrm{~nm})$ 
indicates the range of experimental measurements. ${ }^{1}$ It is clear that such measurements are strongly affected by finite size effects. Figure $5 \mathrm{~d}$ details $R_{\mathrm{g}} / R_{\mathrm{h}}$ for chains with contour lengths given by the experimental molecular weight distribution range. ${ }^{1}$ Here, we take into account three distributions representing polyphenylene precursors samples, prepared under different conditions, after fractionation. ${ }^{1}$ Vertical lines, solid pink, dotted green, and dashed blue, represent values of $L$ obtained from the corresponding weight average molecular weight, $M_{\mathrm{W}}$, of the distributions. Moreover, our results suggest that chains with different compositions in this experimental range have virtually the same $R_{\mathrm{g}} / R_{\mathrm{h}}$. From these observations, we argue that this ratio by itself is not an appropriate parameter to characterize the short chains considered in experiments because of the finite size effects present but also because it brings the same results for clearly different chain types (see the discussion below about persistence length ratios).

Experimentally, light scattering measurements provide additional information about the value of $R_{\mathrm{g}} / R_{\mathrm{h}}$. In particular, dynamic light scattering allows us to obtain the diffusion coefficient $D$ of a polymer coil in a solution with viscosity $\eta$. Through the Stokes-Einstein relation, $D=\frac{k_{\mathrm{B}} T}{4 \pi \eta R_{\mathrm{h}}}$, the radius $R_{\mathrm{h}}$ of the polymer coil is obtained. Dynamic light scattering of two isomeric mixtures, namely, $2-\mathrm{I}\left(M_{\mathrm{W}}=108 \pm 8 \mathrm{~kg} / \mathrm{mol}\right)$ and 2 -II $\left(M_{\mathrm{W}}=470 \pm 30 \mathrm{~kg} / \mathrm{mol}\right)$, of monomers depicted in Figure 1 has been carried out; ${ }^{1,7}$ however, different ways of calculating $D$ lead to rather distinct values of $R_{\mathrm{h}}$. Measurements of $D$ consider the Yamakawa-Fujii theory of monodisperse worm-like chains and produce $R_{\mathrm{g}} / R_{\mathrm{h}}$ ratios systematically smaller than those obtained with methods that include polydispersity effects. ${ }^{14}$ Experimentally, $R_{\mathrm{g}} / R_{\mathrm{h}}^{2-\mathrm{I}, 2-\mathrm{II}}=1.1428,1.3104^{1}$ for chain lengths $L_{\exp }^{2-\mathrm{I}}=$ $130 \mathrm{~nm}$ and $L_{\text {exp }}^{2-I I}=565 \mathrm{~nm}$. The experimental contour length is computed as $L_{\exp }=\left(\frac{M_{\mathrm{W}}}{M_{\text {mon }}}\right) l_{\text {mon }}$, with monomer length $l_{\text {mon }}^{m, p}=$ $0.86 \mathrm{~nm}$ as defined in the present work. $M_{\mathrm{W}}$ and $M_{\text {mon }}=717 \mathrm{~g} /$ mol correspond to the polymer and monomer molar masses, respectively. Experimentally, the radius of gyration is calculated through its dependence on chain and persistence lengths in the worm-like model. ${ }^{15}$ Our random walk chains reach much larger lengths than those experimentally achieved, and we observe that for simulated chains, with $L>8 \times 10^{3} \mathrm{~nm}$, size effects are no longer relevant. As mentioned before, experimental characteristic ratios are in the region where finite size effects are important for our simulated chains.

The effective length of the equivalent Kuhn segment, $l_{\mathrm{k}}=2 l_{\mathrm{p}}$, is calculated from the ratio $l_{\mathrm{k}}=\left\langle R_{\mathrm{e}}^{2}\right\rangle / L$, where $L$ is determined by the average bond length between two beads, $l_{b}$, and the number of segments, $N\left(L=l_{\mathrm{b}} N\right)$. This is in contrast to the definition of the chemical contour length used by the experimentalist, who compute $L$ by taking the monomer length times the number of monomers. Figure 6 shows the persistence length for the different single type polymers and for copolymers of different compositions. Ratios between persistence lengths $l_{\mathrm{p}}^{(x)} / l_{\mathrm{p}}^{m}-$ poly are included taking as a reference the homopolymer $m$-poly. Even for only a few hundred of monomers, we are in the asymptotic limit based on $R_{\mathrm{e}}$ or $R_{\mathrm{g}}$. A lateral extended version (Figure 1c) of the systems considered here has also been synthesized, ${ }^{16}$ and since the backbone of these polyphenylene precursors is the same, our results can be applied to the lateral extended versions as well. A similar behavior of the persistence length is expected since the form of the torsional potential in Figure 2 will not change significantly by changing the size of the

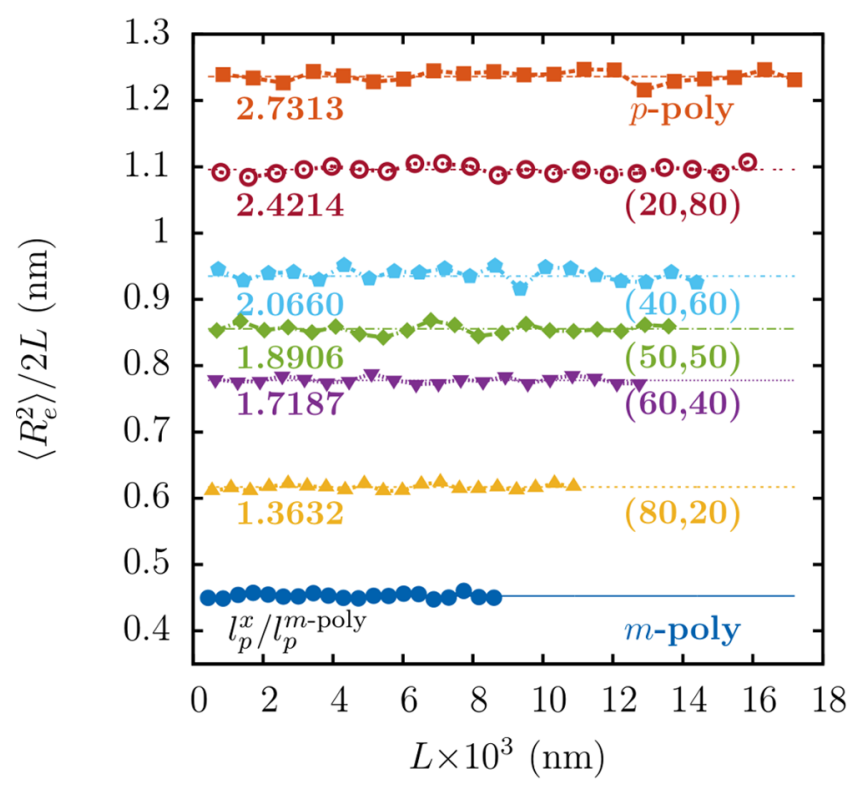

Figure 6. Mean square end-to-end distance, $\left\langle R_{\mathrm{e}}^{2}\right\rangle / L$, as a function of the contour length, $L=l_{\mathrm{b}} N$. The inset numbers correspond to the ratio between persistence lengths, $l_{\mathrm{p}}^{(x)} / l_{\mathrm{p}}^{m-\text { poly }}$. Copolymers persistence lengths lie between the two homopolymer cases.

side groups. Then, the backbone will contribute in the same way. The stiffness of chains with a long average bond length $l_{b}$ increases. Statistically, chains made by para-substituted monomers are more rigid than those with a higher presence of a meta substitution pattern. In the limit of these chain lengths, our analytic prediction of the $l_{\mathrm{p}}^{(x)} / l_{\mathrm{p}}^{m}-$ poly ratio is obtained with respect to composition. Figure 7 presents the persistence length ratios for randomly organized chains at different mixing ratios.

Until now, we have taken into account only the dependence of the persistence length on chain composition. Figure 8 presents the persistence length ratio behavior for different chain compositions as a function of monomer sequence for the largest

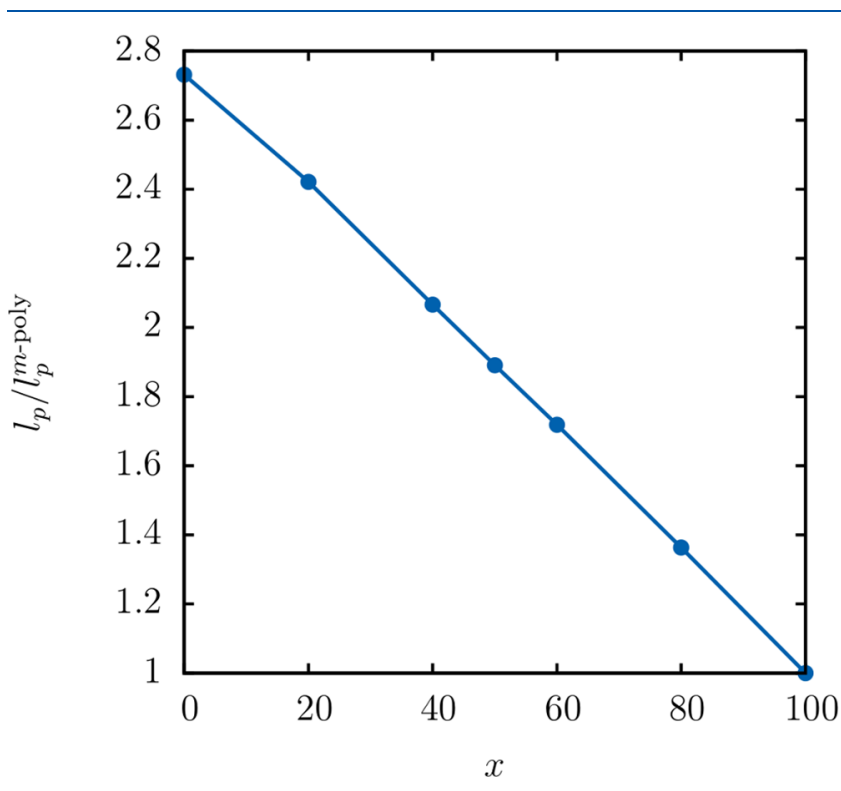

Figure 7. Persistence length ratios for randomly organized chains with different compositions $((x, y)=\{(20,80),(40,60),(50,50),(60,40)$, $(80,20))$. 


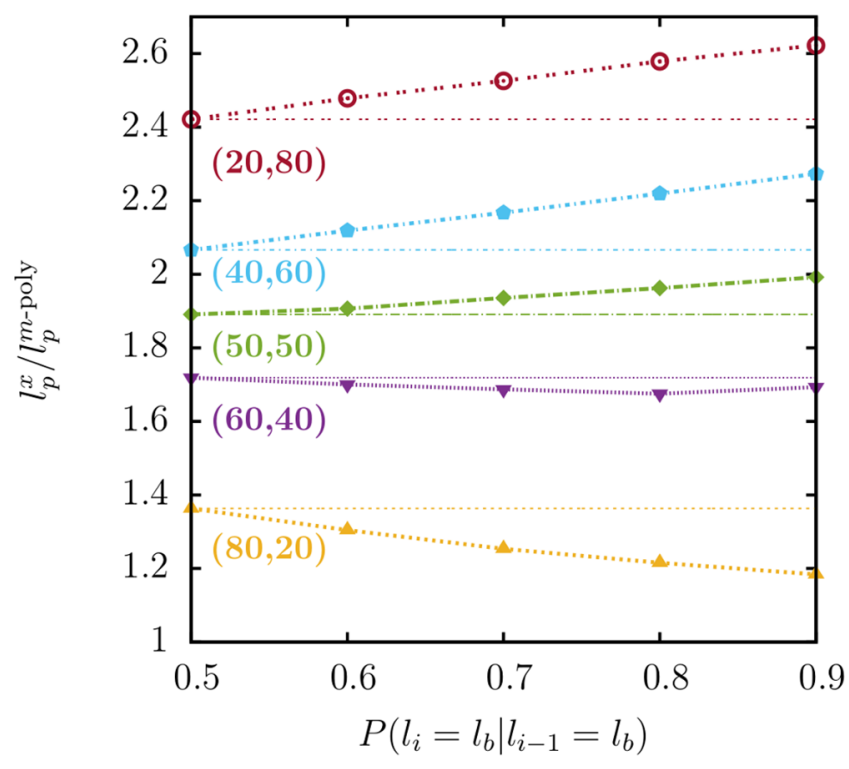

Figure 8. Persistence length ratios for different chain compositions as a function of the conditional probability. The case when $P\left(l_{i}=l_{\mathrm{b}} \mid l_{i-1}=\right.$ $\left.l_{\mathrm{b}}\right)=0.5$ corresponds to the randomly organized cases shown in Figure 4.

chain length in each case. This sequence is given by the conditional probability $P\left(l_{i}=l_{\mathrm{b}} \mid l_{i-1}=l_{\mathrm{b}}\right)$ that $l_{i}=l_{\mathrm{b}}$ given that $l_{i-1}=l_{\mathrm{b}}$. For a given chain fraction, $P\left(l_{i}=l_{\mathrm{b}} \mid l_{i-1}=l_{\mathrm{b}}\right)$ goes from 0.5 to 0.9 . Clearly, a value of 0.5 means that the two monomer orientations and therefore, the two different bond lengths associated to them are randomly organized, that is, the bonds while building the chain do not depend on the previous bond and are only given by their global fraction.

On the other hand, a value of 0.9 corresponds to a higher probability of having the same substitution pattern as the preceding monomer, producing, in that way, an ordered sequence of monomers. A simple way to quantify the ordering along the chain is to look at the distribution of the number of equal segments together $(z)$ along the chain normalized with respect to the chain number of bonds (inset in Figure 9). This distribution is proportional to $\lambda \exp (-\lambda z)$, where $1 / \lambda$ is associated to the mean value of the number of segments together. Figure 9 shows $1 / \lambda$ as a function of the mixing percentage of the $m$-system monomer for each conditional probability. For a given composition, as the conditional probability favors the same types of monomers, a block pattern arises as evidenced by an increase in the $1 / \lambda$ value. As expected, larger blocks are observed when a given type of monomer is in a higher percentage with respect to the other, and the probability of having the same monomer type is high. The contour length is fixed by the composition, but the mean square end-to-end distance differs for the different sequences, and therefore, the calculated persistence lengths also vary. Compositions favoring meta-substituted monomers present a decrease in their persistence length ratios, while chains mainly composed of para-substituted monomers show an increase. The sequence of monomers affect the flexibility of the chain, and the stiffness of the chain raises when blocked polymers are formed with mainly para-substituted monomers.

Experimental characterization of these polyphenylene precursors needs to involve the determination of $R_{\mathrm{g}} / R_{\mathrm{h}}$ and the persistence length. However, when considering the ever present backbone effect, we show that in the experimental range, in

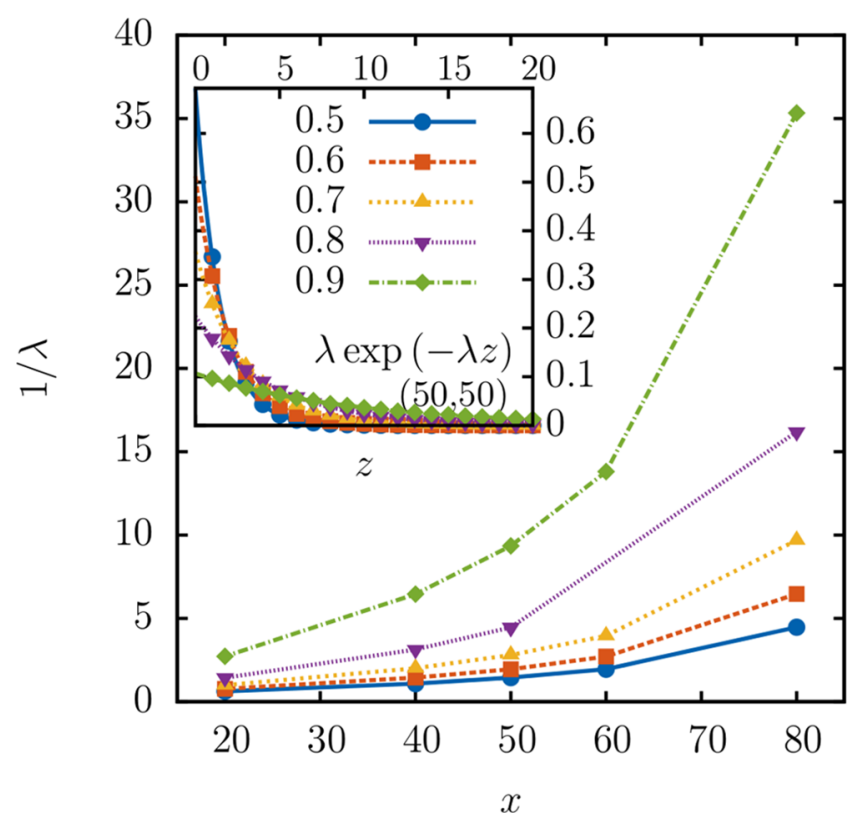

Figure 9. Mean value of number of segments together $(z)$ along the chain as a function of chain composition $x$ for each conditional probability. As the conditional probability favors the same type of monomers, a block pattern arises as it is verified by an increase in the $1 /$ $\lambda$ value. The inset plot shows the probability distribution for $(50,50)$ chain composition and different conditional probabilities.

addition to size effects, composition and monomer sequence also affect these characteristic quantities. Therefore, one should keep monomer connection patterns in mind when designing GNR precursors.

Surface Projections. The final size of synthesized GNRs depends on the lengths of the precursors reached in the polymerization process. Whether the GNRs are solution synthesized or their preparation is mediated by a metallic surface, the final objective of both bottom-up techniques is to produce ribbons of significant length to be deposited onto substrates for device fabrication. Deposition of GNRs onto insulating substrates is still a matter of investigation, either by depositing GNR dispersions ${ }^{4,5}$ or by full layer transfer. ${ }^{6}$ Experimentally, statistical analysis of individual deposited GNR lengths provides an indirect measurement of the reachable precursor sizes, which cannot be obtained by direct analysis on the precursors. ${ }^{1,5}$ These solution-synthesized precursors are subjected to the cyclodehydrogenation in solution, which maintains the aggregation problems and the difficulty in the deposition. Hence, it would be ideal to "graphitize" the solutionmade precursors on the surface to make flat GNRs with long lengths, which unfortunately cannot yet be achieved by the pure on-surface synthesis. In this context, in what follows, we look first at a simplified approximation of the situation of a chain approaching a surface and being adsorbed instantaneously as if projected onto that surface. Straight portions of chains are related to the experimentally reachable precursor length. A first approximation to investigate how the size of the precursors is affected by different monomer sequences and composition of the chain is done by looking at the $2 \mathrm{D}$ projections of those chains onto a plane. Figure 10 shows a schematic view of a projected single chain onto one of the Cartesian planes.

We present a geometric measure of the number of interchain crossings observed when a chain is projected onto a plane. We compute the average density of number of crossings (DNC $=$ 


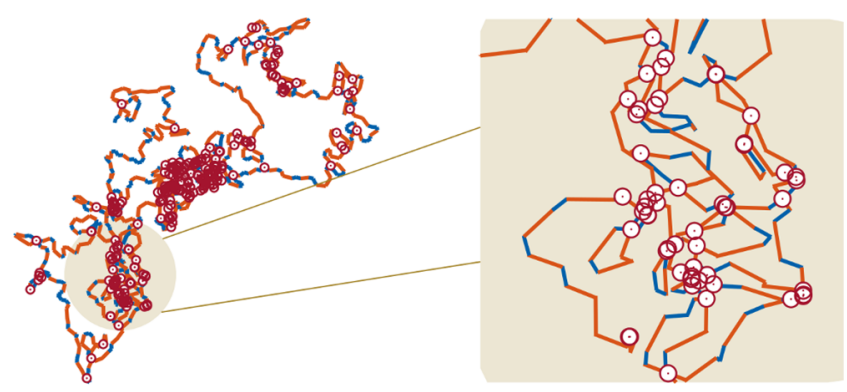

Projection on $x y$ plane

Figure 10. Schematic view of a 1000 beads single chain projected onto the $x y$-plane. Red open symbols denote the crossings while different colors (online) correspond to projected segments of the $m$-poly (blue) or $p$-poly (orange) types. Lengths of segments without crossings may in principle be related to GNR lengths when being deposited onto a surface.

number of crossings $/ L$ ) for the projection onto the three planes defined by the three Cartesian axes. To calculate crossings in a plane, we use the $2 \mathrm{D}$ adaptation of the $3 \mathrm{D}$ line intersection algorithm ${ }^{17}$ as presented in the Supporting Information.

Figure 11 shows the number of crossings averaged over the projection onto the three planes for three different compositions and five selected chain lengths. Homopolymer cases are also included in Figure 11a,c. We observe that for a given composition, DNC values increase with chain length. Eventually, these values have to reach a constant since the fractal dimension of a random walk is $d_{\mathrm{F}}=2$. However, we observe different behaviors with respect to the sequence of monomers. For chains mainly composed of meta-substituted monomers (Figure 11a), DNC increases monotonically with increasing sequence probability, while it decreases for chains made mostly of para-substituted monomers (Figure 11c). When equal mixture ratios are used (Figure 11b), DNC keeps an almost constant value, independent of the chain sequence.

Figure 12 presents the distributions of projected segment lengths (PSL) of the polyphenylene precursors without crossings (not necessarily straight segments) for three different chain compositions and conditional probabilities. In the calculation of the crossings, we assign vectors between the $i$ th and $(i+1)$ th and the $k$ th and $(k+1)$ th beads and check whether the projections of those two vectors intercept. If a given projected vector does not cross with another one, the projected distance between two beads contributes to the segment length value. However, when the projected vector crosses with any other vectors and given that there could be more than one crossing per vector, we take only one crossing point (the average between all crossing points within the same vector) to calculate the segment length and therefore to avoid accounting for lengths within the same projected vector. Here, all plots correspond to chains with $N$ close to 1000 beads. We observe that for a given composition, the maxima of the PSL distributions appear to be independent of the sequence of monomers (Figure 12a). Moreover, when looking at a given chain sequence with either a randomly organized, $P\left(l_{i}=l_{\mathrm{b}} \mid l_{i-1}=l_{\mathrm{b}}\right)=0.5$, or imposed monomer sequence, $P\left(l_{i}=l_{\mathrm{b}} \mid l_{i-1}=l_{\mathrm{b}}\right)=0.8$, there is a clear shift in the maxima positions of the distributions for different chain compositions. Despite the increase in the width of the distribution as a function of chain stiffness, we observe on average short projected segments between 0.5 and $1.0 \mathrm{~nm}$. Nevertheless, longer segments exist in all cases (Figure 12b), and it should be possible to generate long GNRs by tuning the

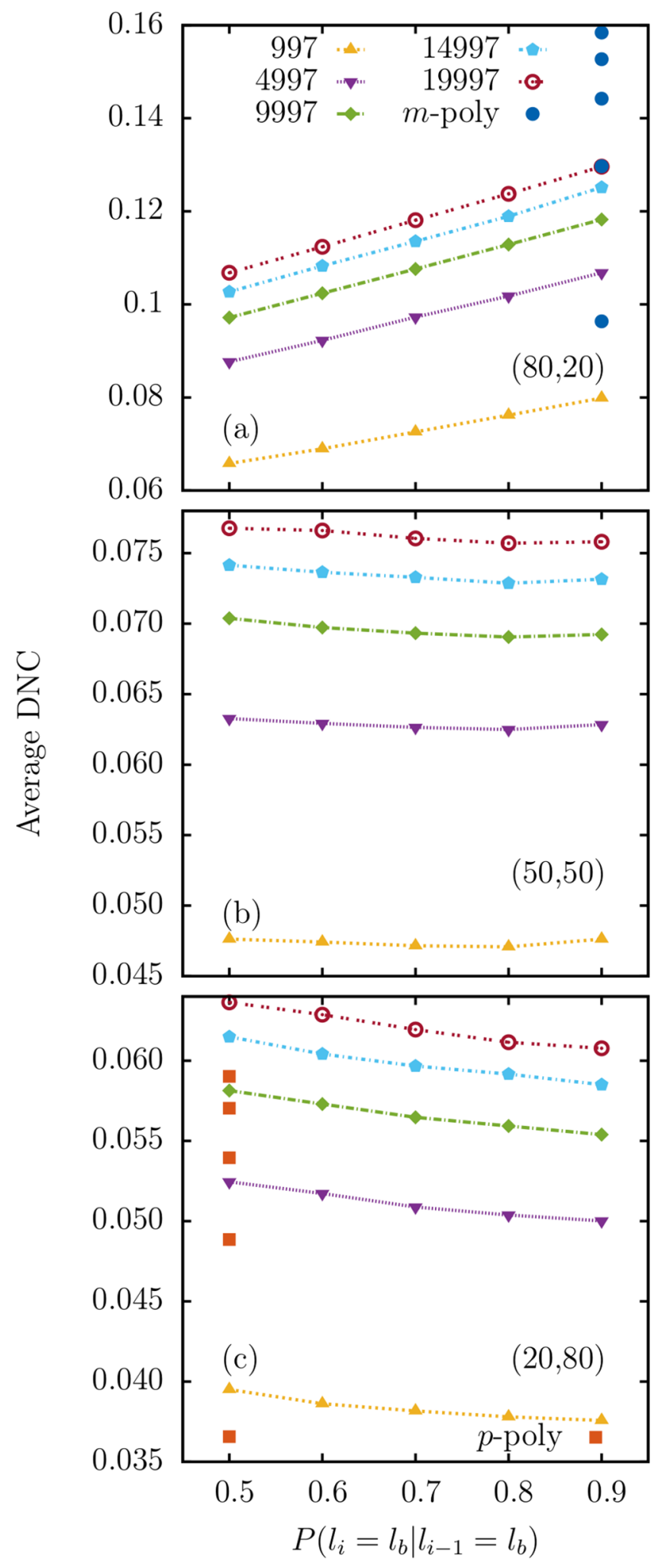

Figure 11. Average density number of crossings as a function of the conditional probability $P\left(l_{i}=l_{\mathrm{b}} \mid l_{i-1}=l_{\mathrm{b}}\right)$ for three different chain compositions and selected number of segments. (a and c) include DNC values in the case of homopolymers $m$-poly (blue solid circles) and $p$ poly (orange solid squares).

strength of the interaction energy between the surface and the polymer chains. 


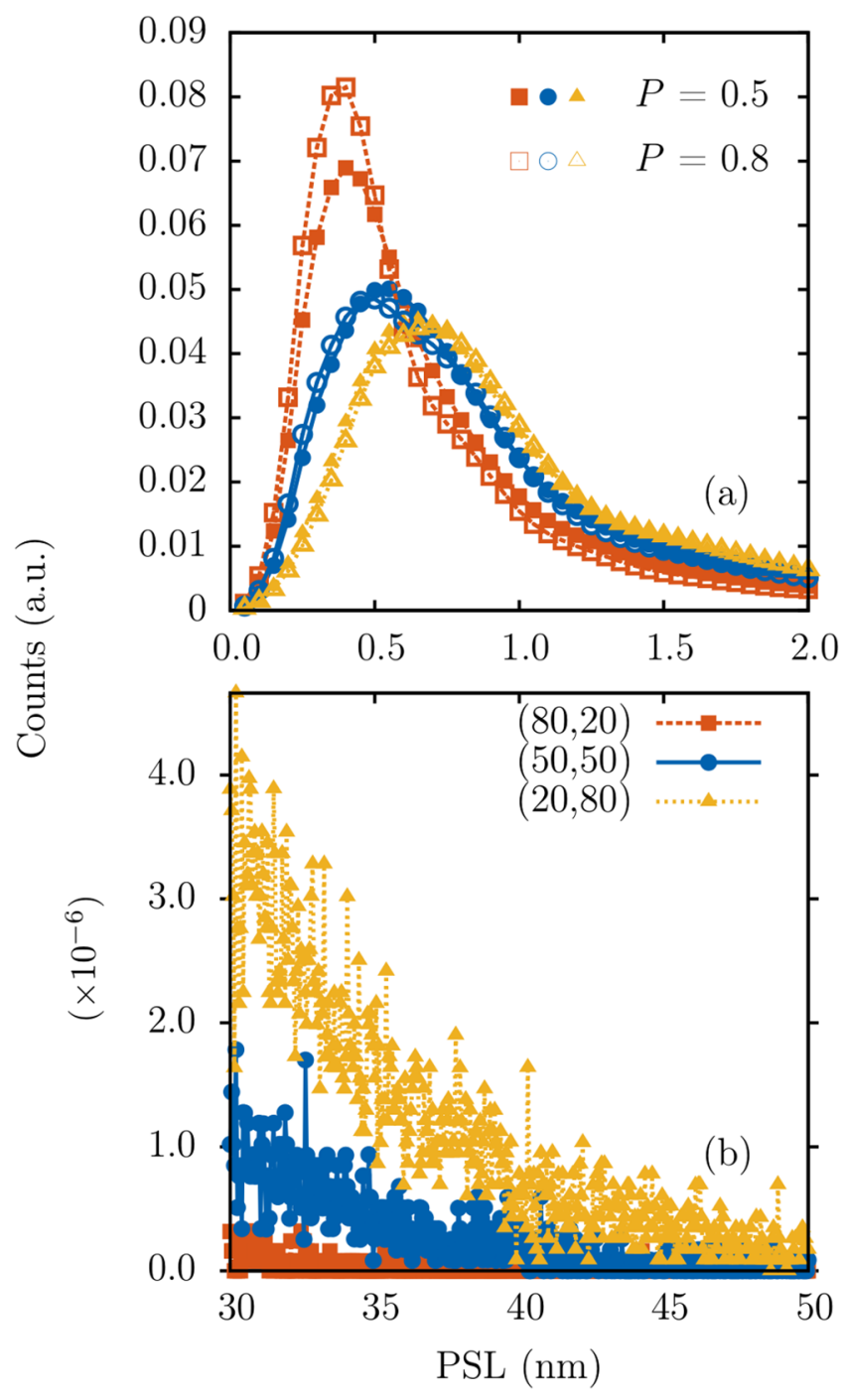

Figure 12. Distribution of projected segment lengths (PSL) without crossings for (a) three different compositions and two conditional probabilites $\left(P\left(l_{i}=l_{\mathrm{b}} \mid l_{i-1}=l_{\mathrm{b}}\right)\right), P=0.5$ (solid symbols) $P=0.8$ (open symbols) and (b) counts for PSLs up to $50 \mathrm{~nm}$ with $P=0.5$. These counts are less significant than shorter segment lengths.

Chemical composition and sequence probability affect the length of the polymer precursors and therefore are expected to affect the size of the produced GNRs. A low density number of crossings is then related to long polyphenylene segments that, in turn, produce long GNRs. Then, from our analysis, long GNRs are mainly the result of polymer precursors made mainly of parasubstituted monomers and the optimized chain length.

\section{CONCLUSIONS}

Experimental characterization of polyphenylene precursors with distinct molecular weights gives unexpectedly different, that is, semirigid to semiflexible, structural behaviors. In this paper, we considered the backbone contributions to the persistence length in an ideal chain model. We demonstrated how structural changes influence the persistence lengths. These changes are a consequence of having polymers constituted by the same precursor monomer but exhibiting positional isomerism. Ideal polymer chains representing polyphenylene GNR precursors were constructed by taking into account the nature of the arene substitutions along the polymer backbone, thus isolating the always present steric backbone effects. Persistence length ratios of a polymer facilitate a comparison between theoretical and experimental studies. The behavior of relative values of persistence lengths with respect to the $m$-substitution limiting case gives a direct description of the chain flexibility. We found that chain composition and monomer sequence affect the stiffness of a chain. We showed that persistence length ratios for different chain compositions and conditional bonding probabilities are substantially different. Since the persistence length, which is well defined only for random walks, is an intrinsic property of the chemical structure of the polymer, it is not expected to depend on the molecular weight well above $L / l_{\mathrm{p}}=$ $1.9,18$ This result indicates that the $l_{\mathrm{p}}$ values experimentally obtained do not reveal the changing structure of the polyphenylene precursors and lead to inadequate interpretation of the experimental results. Moreover, we emphasized that experimental measurements ${ }^{1,7}$ of $R_{\mathrm{g}} / R_{\mathrm{h}}$ ratios are strongly affected by finite size effects and that in addition to $R_{\mathrm{g}} / R_{\mathrm{h}}$ and $l_{\mathrm{p}}$, a parameter accounting for composition and monomer sequence of the polymer is necessary to precisely characterize these types of polyphenylene precursors.

Long-length GNRs are the result of the cyclodehydrogenation of solution-synthesized precursors in solution, which leads to the known problems of aggregation and difficulty in deposition. An ideal scenario would be a cyclodehydrogenation of the solutionmade precursors on the surface to produce flat GNRs. In this context, the average $2 \mathrm{D}$ density of the number of crossings of the resulting polymer chains represents a simplified approximation of this ideal case and therefore is also related to the deposition of GNRs onto insulated substrates. Chemical composition and monomer ordering affect the length and size of the produced GNRs. As a result, a low density of crossings relates unequivocally to long polyphenylene segments and thus to long GNRs. Statistically, preferably long GNRs result from a combination of both a high fraction of para-substituted monomers and a large monomer sequence probability, namely, by more stiffer polyphenylene precursors.

\section{ASSOCIATED CONTENT}

\section{Supporting Information}

The Supporting Information is available free of charge on the ACS Publications website at DOI: 10.1021/acs.macromol.9b00819.

Description of how the conditional probability is taken into account and the calculation of crossings and contributions to the total ab inito energy curve (PDF)

Movie showing the single point optimized geometries during the scaning of the dihedral angle (MP4)

\section{AUTHOR INFORMATION}

\section{Corresponding Authors}

*E-mail: forero@mpip-mainz.mpg.de (N.C.F-M.).

*E-mail: kremer@mpip-mainz.mpg.de (K.K.).

\section{ORCID}

Nancy C. Forero-Martinez: 0000-0001-8903-7878

Björn Baumeier: 0000-0002-6077-0467

Kurt Kremer: 0000-0003-1842-9369

\section{Notes}

The authors declare no competing financial interest. 


\section{ACKNOWLEDGMENTS}

This work has been supported by the European Research Council under the European Union's Seventh Framework Programme (FP7/2007-2013)/ERC Grant Agreement No. 340906-MOLPROCOMP. We thank Akimitsu Narita, George Fytas, Cristina Greco, and Hsiao-Ping Hsu for stimulating discussion. We are greatful to Robinson Cortes-Huerto, Jan Smrek, and Akimitsu Narita for a critical reading of the manuscript.

\section{REFERENCES}

(1) Narita, A.; et al. Synthesis of structurally well-defined and liquidphase-processable graphene nanoribbons. Nat. Chem. 2014, 6, 126132.

(2) Narita, A.; Wang, X.-Y.; Feng, X.; Müllen, K. New advances in nanographene chemistry. Chem. Soc. Rev. 2015, 44, 6616-6643.

(3) Cai, J.; Ruffieux, P.; Jaafar, R.; Bieri, M.; Braun, T.; Blankenburg, S.; Muoth, M.; Seitsonen, A. P.; Saleh, M.; Feng, X.; Müllen, K.; Fasel, R. Atomically precise bottom-up fabrication of graphene nanoribbons. Nature 2010, 466, 470-473.

(4) Abbas, A. N.; Liu, G.; Narita, A.; Orosco, M.; Feng, X.; Müllen, K.; Zhou, C. Deposition, Characterization, and Thin-Film-Based Chemical Sensing of Ultra-long Chemically Synthesized Graphene Nanoribbons. J. Am. Chem. Soc. 2014, 136, 7555-7558.

(5) Konnerth, R.; Cervetti, C.; Narita, A.; Feng, X.; Müllen, K.; Hoyer, A.; Burghard, M.; Kern, K.; Dressel, M.; Bogani, L. Tuning the deposition of molecular graphene nanoribbons by surface functionalization. Nanoscale 2015, 7, 12807-12811.

(6) Bennett, P. B.; Pedramrazi, Z.; Madani, A.; Chen, Y.-C.; de Oteyza, D. G.; Chen, C.; Fischer, F. R.; Crommie, M. F.; Bokor, J. Bottom-up graphene nanoribbon field-effect transistors. Appl. Phys. Lett. 2013, 103, 253114.

(7) Wunderlich, K.; Müllen, K.; Fytas, G. Conjugated Polymers and Oligomers; World Scientific: 2018; Chapter 1, pp 1-28.

(8) Rubinstein, M.; Colby, R. Polymer Physics; Oxford University Press: Oxford, 2003

(9) Flory, P.; Jackson, J. Statistical Mechanics of Chain Molecules; Hanser: 1989.

(10) Frisch, M. J. et al. Gaussian 09 Revision E.01. Gaussian Inc. Wallingford, CT 2009.

(11) Teraoka, I. Polymer Solutions; John Wiley \& Sons, Inc.: 2002, pp 167-275.

(12) Dünweg, B.; Reith, D.; Steinhauser, M.; Kremer, K. Corrections to scaling in the hydrodynamic properties of dilute polymer solutions. J. Chem. Phys. 2002, 117, 914-924.

(13) Clisby, N.; Dünweg, B. High-precision estimate of the hydrodynamic radius for selfavoiding walks. Phys. Rev. E 2016, 94, No. 052102 .

(14) Harnau, L.; Winkler, R. G.; Reineker, P. Influence of Polydispersity on the Dynamic Structure Factor of Macromolecules in Dilute Solution. Macromolecules 1999, 32, 5956-5960.

(15) Pedersen, J. S.; Schurtenberger, P. Scattering Functions of Semiflexible Polymers with and without Excluded Volume Effects. Macromolecules 1996, 29, 7602-7612.

(16) Narita, A.; Verzhbitskiy, I. A.; Frederickx, W.; Mali, K. S.; Jensen, S. A.; Hansen, M. R.; Bonn, M.; de Feyter, S.; Casiraghi, C.; Feng, X.; Müllen, K. Bottom-Up Synthesis of Liquid-Phase-Processable Graphene Nanoribbons with Near-Infrared Absorption. ACS Nano 2014, 8 , 11622-11630.

(17) Goldman, R. Graphics Gems; Glassner, A. S., Ed.; Academic Press Professional, Inc.: 1990, pp 304-305

(18) This statement holds on the level of accuracy that can be obtained experimentally in this context. A recent detailed critique of the persistence length concept is beyond the present approach. ${ }^{19}$

(19) Binder, K.; Hsu, H.-P.; Paul, W. Understanding the stiffness of macromolecules: From linear chains to bottle-brushes. Eur. Phys. J. Spec. Top. 2016, 225, 1663-1671. 